\title{
Indicators of space weather events in cosmic rays during the solar cycle 24
}

\section{Agnieszka Gil*}

Siedlce University, Institute of Mathematics and Physics, Siedlce, Poland; Space Research Center of Polish Academy of Sciences, Warsaw, Poland

E-mail: gila@uph.edu.pl

\section{R. Modzelewska}

Siedlce University, Institute of Mathematics and Physics, Siedlce, Poland

\section{Sz. Moskwa}

Department of Electrical and Power Engineering, AGH University of Science and Technology, Krakow, Poland

\section{A. Siluszyk}

Siedlce University, Institute of Mathematics and Physics, Siedlce, Poland

\section{Siluszyk}

Siedlce University, Institute of Mathematics and Physics, Siedlce, Poland

\section{A. Wawrzynczak}

Siedlce University, Institute of Computer Sciences, Siedlce, Poland

Solar originating events are repeatedly observable in cosmic ray flux measured at the ground by neutron monitors. We analyze time intervals of halo coronal mass ejections (CMEs), as well as sudden storm commencements (SSCs) appearance during solar cycle 24. We consider neutron monitor data, as well as, solar, heliospheric and geomagnetic activity parameters, around those periods, using different mathematical tools. Our computations show that the fractal dimension calculated for each week of studied period, based on Oulu neutron monitor hourly data, decreases around those events. Skewness and kurtosis change abruptly from the background average value. Since an influence of space weather phenomena on energy infrastructure is well known, in the next step we consider a list of electrical grids failures which occurred in southern Poland during the studied time intervals of halo CMEs and SSCs occurrence. Based on the data from Institute of Meteorology and Water Management-Polish National Research Institute we exclude from the analysis failures caused by the meteorological effects.

36th International Cosmic Ray Conference -ICRC2019-

July 24th-August 1st, 2019

Madison, WI, U.S.A.

\footnotetext{
*Speaker.
} 


\section{Introduction}

Earth's environment is affected by the solar-driven phenomena generally known as space weather. This term 'space weather' concerns circumstances on Sun and in the heliosphere that might influence the space born and ground based technological systems, as well as human existence. Variability of Sun is touching the Earth in a number of ways, depending on the level of its activity. Solar activity leads to a transformation of magnetic energy into space-weather-driving effects. In solar maxima transient phenomena as solar flares (SF) and coronal mass ejections- which often cause Forbush decreases, are very frequent, leading to a growth of injection, acceleration, as well as transport of solar energetic particles (SEPs).

It is well known, that strong magnetic storm affects the normal operation of ground-located electrical systems (which is an issue in our paper) and causes breakdowns of satellites and its equipment. During solar minima, the heliospheric magnetic field (HMF) becomes weaker, leading to an enhancement in the level of the galactic cosmic ray (GCR) intensity. GCR intensity is constantly modified as a result of changes (in a wide range of time-scales) on Sun and its magnetic field. Galactic cosmic rays continuously reaching Earth bring information about the state of Sun and the heliosphere. Thus, understanding GCR properties allows discovering properties of Sun and the heliosphere in an indirect way. They also may be helpful in space weather research, as for instance in determining a level of exposure of aircraft crews to ionizing radiation (e.g., [1]). Moreover, might be used in post-factum analysis of geomagnetic storms.

\section{Data, methods and results}

We have gathered data of minor electrical grids failures (EGFs) from southern Poland in 2010 (around minimum of solar activity) and in January-July 2014 (around maximum of solar activity). Assuming the criteria of failure in accordance with the set used by the Distribution System Operator (DSO) when collecting them, statistical classification of failures causes was made. There were 16 different reasons for power cut offs in the network, whose relative appearance in the analyzed year 2010 for the considered area is shown in Fig. 1a. In 2014 there were 29 different reasons for power cut offs in the network, whose relative occurrence in this second considered time interval for the analyzed area is shown in Fig. 2a.

Because the reasons in some cases were similar, so it was possible to place them in certain subgroups. In further analysis, we considered causes of failures classified in 6 main groups (A-F). The first 3 groups (A-C) determined the reasons for disabling the network, which were caused not by 'solar" factors. The sources of these line exclusions were various types of meteorological effects, operational shut-downs and vandalism. The next 3 groups (D-F) determined the causes of failures that could be triggered by space weather effects (Figures $1 \mathrm{~b}$ and $2 \mathrm{~b}$ ). Table 1 summarising Figures 1 and 2 presents that there was a tendency of a dependence between the number of failures that could have its origin on Sun and the level of solar activity. Solar cycle 24 was very peaceful, but periods of enhanced solar and geomagnetic activity could be easily distinguished. We have considered events starting from moderate geomagnetic storms (with geomagnetic index $\mathrm{Kp} \geq 5$ ).

We used for calculation hourly data of GCR intensity measured by Oulu NM, geomagnetic indexes: $\mathrm{Kp}$, Dst, AE, solar and heliospheric parameters: magnetic field strength B, magnetic field Bz com- 


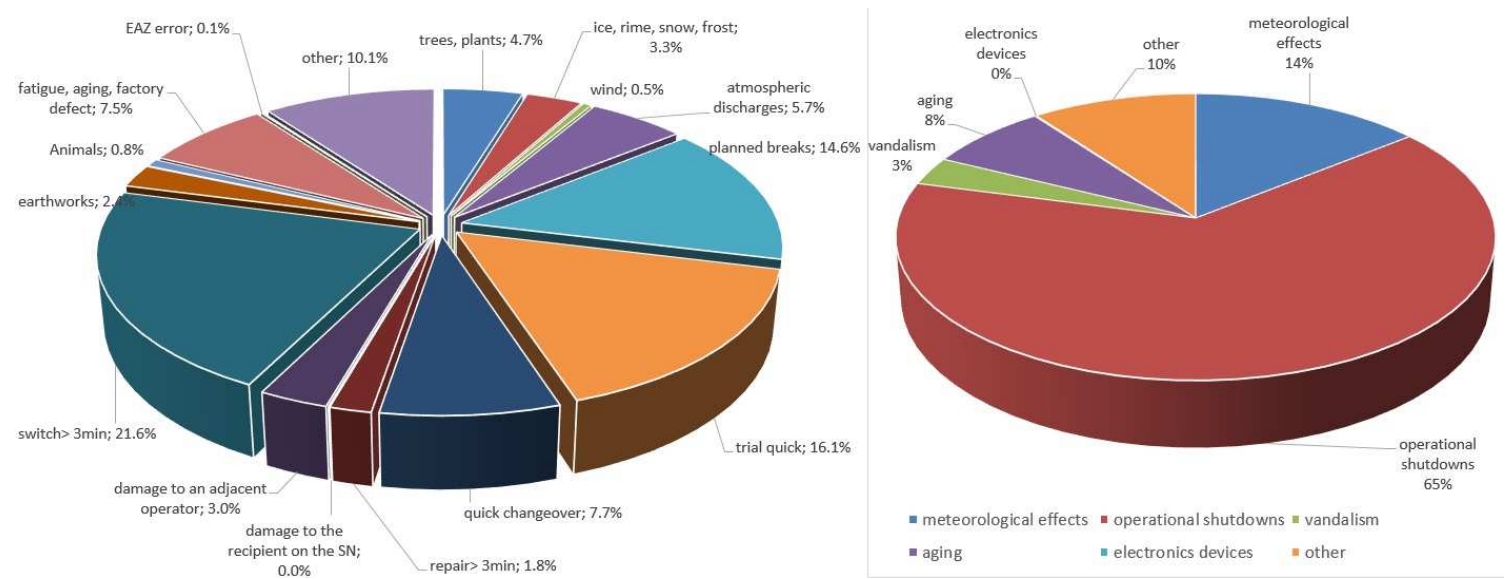

Figure 1: Groups and six classes of the failures causes in 2010
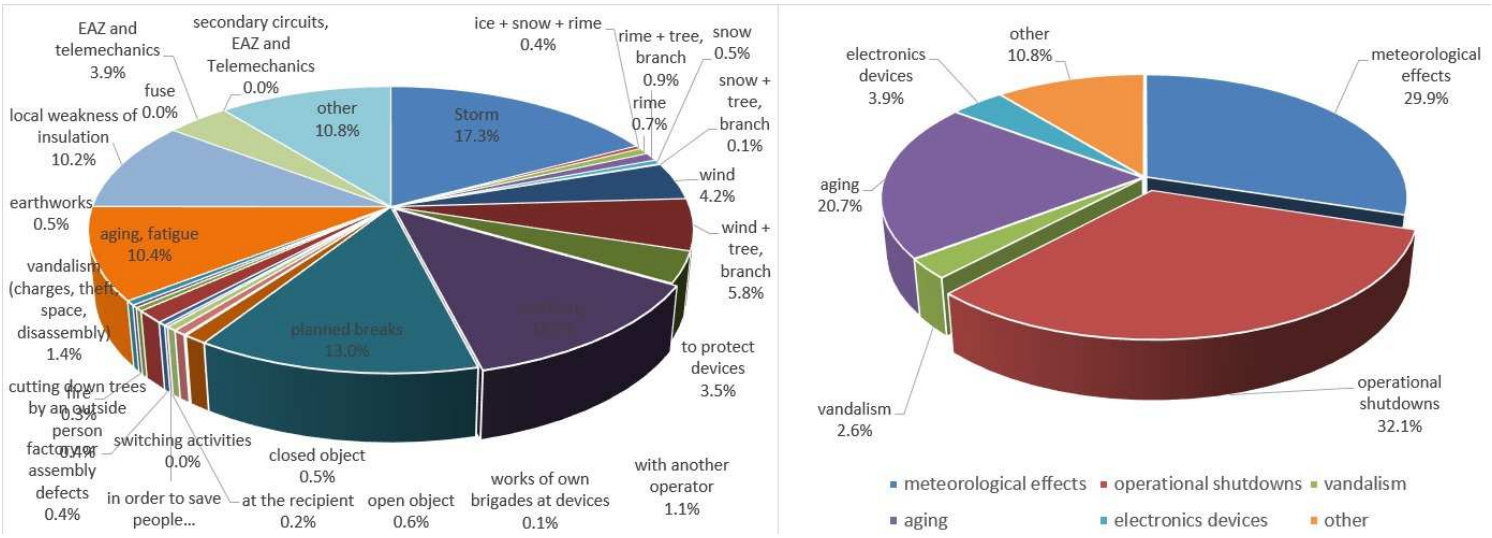

Figure 2: Groups and six classes of the failures causes in 2014

ponent and solar wind speed (SWs) in 2010 and 2014. One of calculated quantities was skewness, being a measure of the distribution's asymmetry. The other was kurtosis, as a measure of the flatness of the distribution. Results of our computations for 2010 year (as an example) present Figure 3 for skewness and Figure 4 for kurtosis. Figures 3-4 display that both, skewness and kurtosis for all of the studied time series behaved steadily, but during the disturbances times changed abruptly. We have calculated also fractal dimension, which displays a time series' complexity. Results are presented on Figure 5 with visible decreases around the space weather events times.

Next, we used IZMIRAN Forbush decreases (Fds) data-base for 2010 and 2014, to extract all the events considered by the IZMIRAN group as Fd.

We took into consideration properties of coronal mass ejecta from CME Catalog - CDAW Data Center ([2]). In 2010 there were registered 1119 CMEs and 2014: 2599. From those we have chosen for further studies only fast halo CMEs. 
Table 1: Electrical grids failures from classes D-F, in 2010 and 2014

\begin{tabular}{|l|l|l|} 
time interval & 2010 & I-VII 2014 \\
\hline \hline total no. of EGFs & 25616 & 30155 \\
\hline EGFs no. caused by the aging of elements & 1917 & 6209 \\
EGFs no. caused by the electronic devices breakdowns & 32 & 1181 \\
EGFs no. having unknown reasons & 2676 & 3266 \\
\hline$\%$ of total EGFs no. & $17.66 \%$ & $35.34 \%$
\end{tabular}

We have considered list of sudden storm commencement from Ebre Observatory. Sudden commencement generally is treated as a precursor of geomagnetic storm [3]. We analyzed events of 20 SSCs: 6 during 2010 and 14 during January-July 2014.

Superposed epoch analysis is a method reviling relationships between the analyzed time series [4]. This tool was formerly used to investigate complex magnetospheric disturbances and peculiarities. Denton et al. [5], examining the correlation of the geomagnetic storm phase with a temporal variation of plasma found at geosynchronous orbit, showed that the one of the key factors for the plasma sheet density is solar cycle. Liemohn et al. [6] studied magnetic storms' features in the relation of time of occurrence, as well as their strength. Ilie et al. [7] analyzed (as a function of local and epoch time) the temporal evolution of geosynchronous plasma parameters. Yermolaev et al. [8] investigated dynamics of large-scale solar wind streams (corotating interaction regions, coronal mass ejections, sheath and interplanetary shocks).

The above mentioned events: when Kp geomagnetic index value was not less than 5, days of Fds, sudden storm commencements, as well as fast halo CMEs, served us to define a key-time. Next, we extracted sets of electrical grids failures number having a particular reason (from Classes D-F), ranging from 2 to 5 days, before and after each key-time. Afterwards, we superposed all extracted failures subsets D-F, synchronizing all key-times. Our results show Figure 6. Each panel of Figure 6 reveals that there was an increase in EGFs number from the superposed average value (here as a reference point being equal 0 ) from 1.5 up to 2.5 times around considered key-times.

\section{Summary}

Behaviour of statistical properties of GCR intensity, as well as solar and geomagnetic parameters can be treated as good indicators of space weather events.

Comparing the total number of electrical grids failures near the solar minimum, 2010 and around the solar maximum, I-VII 2014, the number of EGFs is much greater in 2014 than 2010, so it can be a signal of solar cycle dependence.

The increase in the superposed averaged number of EGF appears around Forbush decreases, SSC and increased $\mathrm{Kp}$-index value above 5, as well as one day after the fast halo CME occurrence.

\section{Acknowledgements}

The Institute of Meteorology and Water Management -National Research Institute for the meteorological data. Data of solar and geomagnetic indexes are from https://omniweb.gsfc.nasa.gov/, 


\section{skewness}

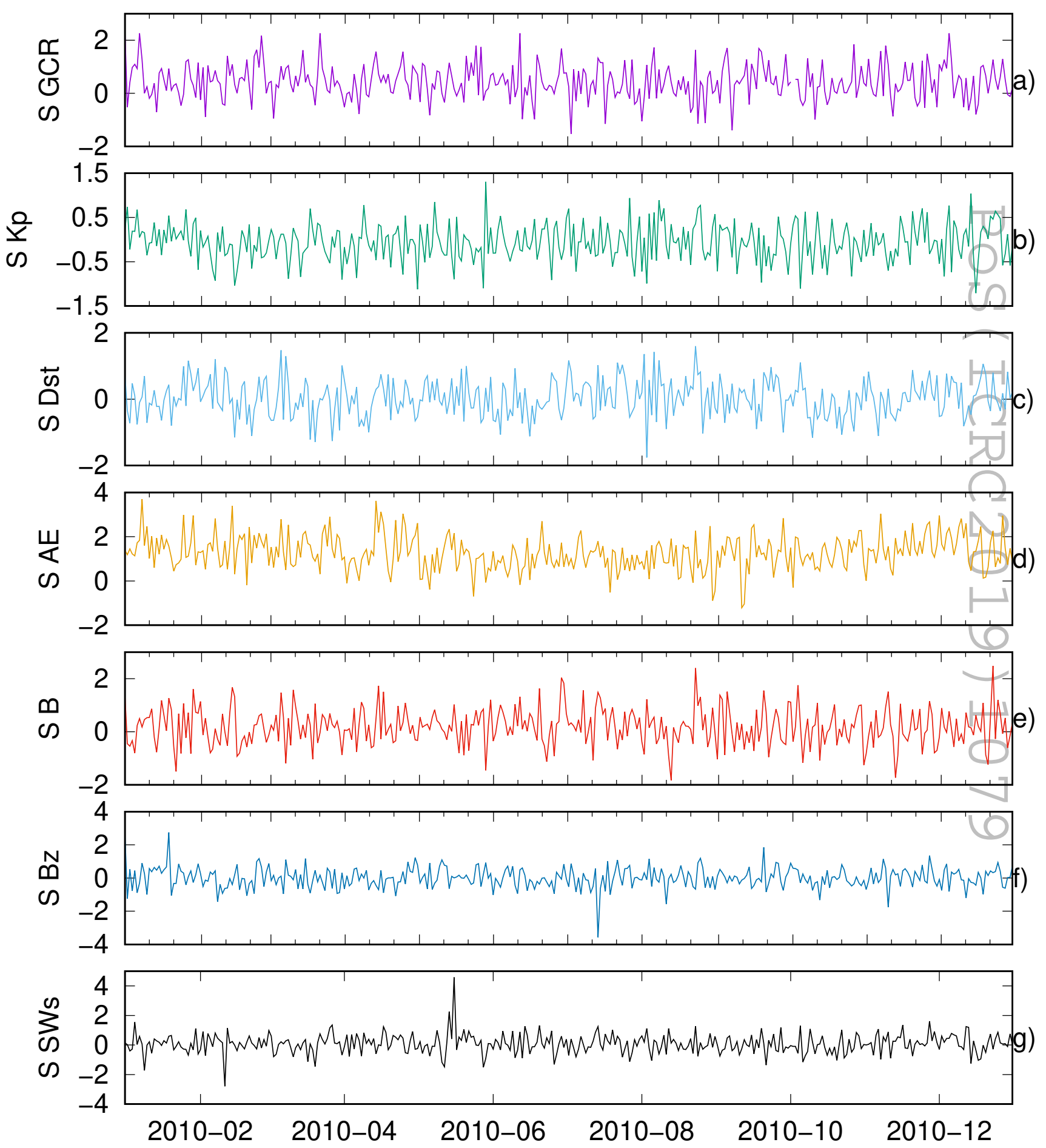

Figure 3: Skewness (S) of hourly data from: (a) GCR intensity measured by Oulu NM; geomagnetic indexes: (b) Kp, (c) Dst, (d) AE; solar and heliospheric parameters: (e) magnetic field strength, (f) magnetic field Bz component and $(\mathrm{g})$ solar wind speed in 2010 


\section{kurtosis}
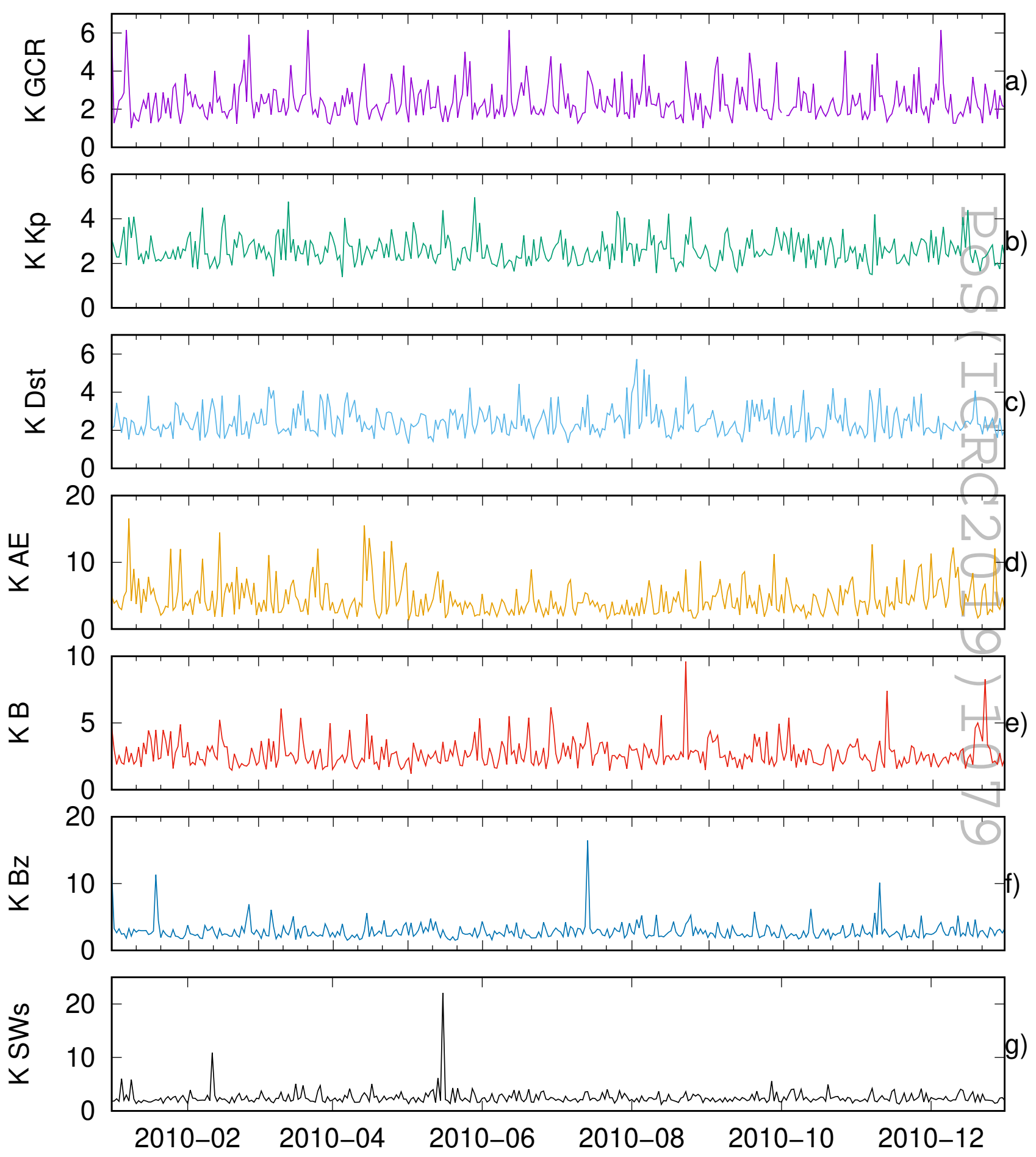

Figure 4: Kurtosis (K) of hourly data from: (a) GCR intensity measured by Oulu NM; geomagnetic indexes: (b) Kp, (c) Dst, (d) AE; solar and heliospheric parameters: (e) magnetic field strength, (f) magnetic field Bz component and $(\mathrm{g})$ solar wind speed in 2010 


\section{fractal dimension}
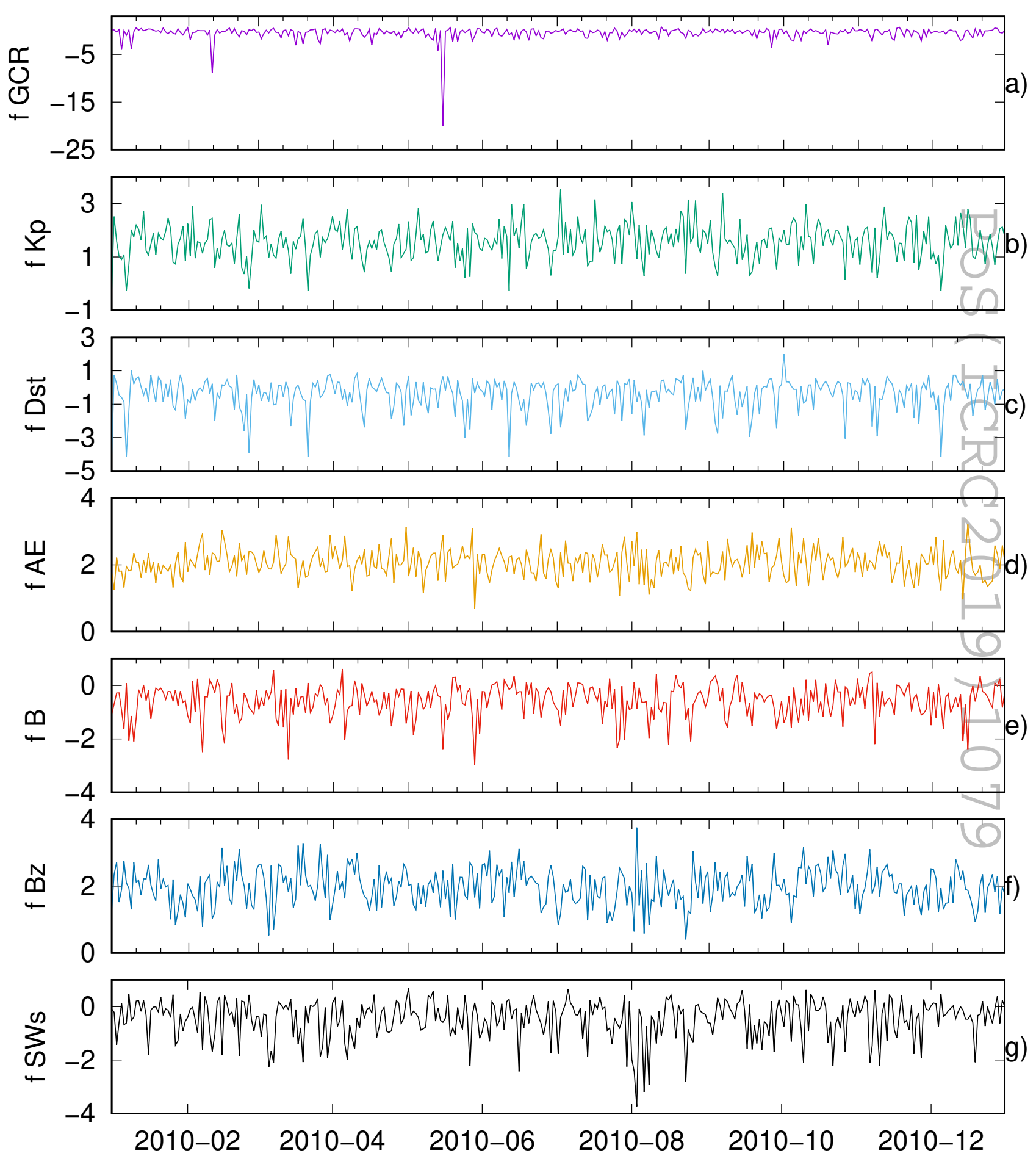

Figure 5: Fractal dimension (f) for hourly data from: (a) GCR intensity measured by Oulu NM; geomagnetic indexes: (b) Kp, (c) Dst, (d) AE; solar and heliospheric parameters: (e) magnetic field strength, (f) magnetic field Bz component and (g) solar wind speed in 2010 

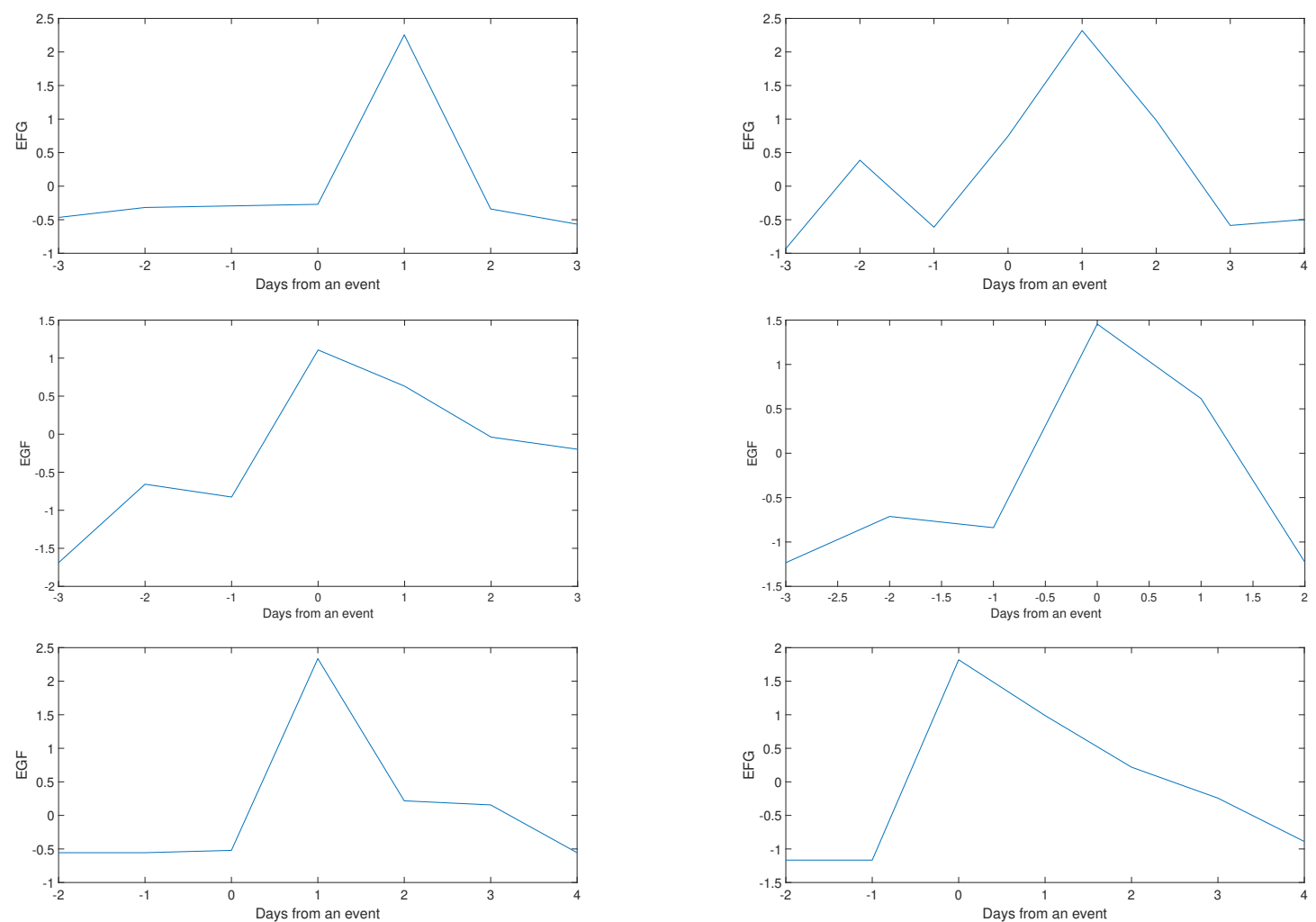

Figure 6: The superposed averaged values for electrical grids failures number (caused by the aging: a, d; electronic devices: b; having unknown reasons: $c, e, f)$, with a key time connected to halo CME occurrence (b, e), growth of Kp index (a), Fd (c, d) and sudden storm commencement (f) in 2014 (a-b) and 2010 (c-f)

cosmic rays from https://cosmicrays.oulu.fi, Fds from http://spaceweather.izmiran.ru/, CME from https://cdaw.gsfc.nasa.gov/ and SSC from http://www.obsebre.es.

We acknowledge the financial support by the Polish National Science Centre, grant no. 2016/22/E/HS5/00406.

Sz. M. and M.S. acknowledge the Polish National Science Centre, grant no. 2014/15/B/ST8/02315

\section{References}

[1] Mishev, A., Poluianov, S., \& Usoskin, I., Journal of Space Weather and Space Climate, 7, A28, 2017

[2] Yashiro S., Gopalswamy N., Michalek G., et al. A catalog of white light coronal mass ejections observed by the SOHO spacecraft, Journal of Geophysical Research, 109, A07105, 2004

[3] Curto, J. J., T. Araki, and L. F. Alberca, Evolution of the concept of Sudden Storm Commencements and their operative identification, Earth Planets Space, 59, I-XII, 2007

[4] Chree, C., Some Phenomena of Sunspots and of Terrestrial Magnetism at Kew Observatory, Philosophical Transactions of the Royal Society of London. Series A, Containing Papers of a Mathematical or Physical Character, 212, 75-116, 1913

[5] Denton, M. H., M. F. Thomsen, H. Korth, S. Lynch, J. C. Zhang, and M. W. Liemohn, Bulk plasma properties at geosynchronous orbit, Journal of Geophysical Research, 110, A07223, 2005 
[6] Liemohn, M. W., J.-C. Zhang, M. F. Thomsen, J. E. Borovsky, J. U. Kozyra, and R. Ilie, Plasma properties of superstorms at geosynchronous orbit: How different are they?, Geophysical Research Letter, 35, L06S06, 2008

[7] Ilie, R., M. W. Liemohn, M. F. Thomsen, J. E. Borovsky, and J. Zhang, Influence of epoch time selection on the results of superposed epoch analysis using ACE and MPA data, Journal of Geophysical Research, 113, A00A14, 2008

[8] Yermolaev, Yu. I., I. G. Lodkina, N. S. Nikolaeva, and M. Yu. Yermolaev, Dynamics of large-scale solar wind streams obtained by the double superposed epoch analysis, Journal of Geophysical Research, 120, 7094-7106, 2015 